\title{
Chloroplast Degradation: Multiple Routes Into the Vacuole
}

\author{
Xiaohong Zhuang ${ }^{1,2 *}$ and Liwen Jiang ${ }^{1,2}$ \\ ${ }^{1}$ School of Life Sciences, Centre for Cell \& Developmental Biology and State Key Laboratory of Agrobiotechnology, The \\ Chinese University of Hong Kong, Hong Kong, China, ${ }^{2}$ The Chinese University of Hong Kong Shenzhen Research Institute, \\ Shenzhen, China
}

Chloroplasts provide energy for all plants by producing sugar during photosynthesis. To adapt to various environmental and developmental cues, plants have developed specific strategies to control chloroplast homeostasis in plant cells, including chloroplast degradation during leaf senescence and the transition of chloroplasts into other types of plastids during the day-night cycle. In recent years, autophagy has emerged as an essential mechanism for selective degradation of chloroplast materials (also known as chlorophagy) in the vacuole. Different types of membrane structures have been implicated to involve in the delivery of distinct chloroplast contents. Here we provide a current overview on chlorophagy and discuss the possible chloroplast receptors and upstream signals in this process.

\section{OPEN ACCESS}

Edited by:

Yuji Moriyasu,

Saitama University, Japan

Reviewed by:

Marinus Pilon,

Colorado State University,

United States

Judy Brusslan,

California State University Long Beach, United States

*Correspondence:

Xiaohong Zhuang

xhzhuang@cuhk.edu.hk

Specialty section: This article was submitted to

Plant Cell Biology,

a section of the journal

Frontiers in Plant Science

Received: 16 December 2018

Accepted: 07 March 2019

Published: 26 March 2019

Citation:

Zhuang X and Jiang L (2019) Chloroplast Degradation: Multiple

Routes Into the Vacuole.

Front. Plant Sci. 10:359,

doi: 10.3389/fp/s.2019.00359
Keywords: chlorophagy, autophagy, vacuole, chloroplast, chloroplast receptor

\section{INTRODUCTION}

The chloroplast, a well-known plastid found in all photosynthetic plant cells, is the central organelle providing plants with foods and energy in the form of sugar or starch by photosynthesis (Jarvis and Lopez-Juez, 2014). Chloroplast turnover plays a critical role in plastid transition (e.g., proplastid to chloroplast) and nutrient mobilization (e.g., carbon and nitrogen) (Siqueira et al., 2018). Upon different stress conditions, chloroplasts may be damaged and produce toxic ROS or stress signals which are detrimental to the plant growth. To cope with a variety of internal or external stresses, plants carry out leaf senescence via selective degradation of chloroplasts to avoid the accumulation of toxic ROS, thus placing a significance of efficient chloroplast turnover under stress conditions (Xie et al., 2015; Izumi and Nakamura, 2018; Nakamura and Izumi, 2018; Otegui, 2018; Soto-Burgos et al., 2018). Recent evidence suggests that chloroplast materials are sequestered into multiple types of subcellular structures for their delivery into the lytic vacuole. Novel insights into our understanding of chloroplast turnover have been obtained by recent studies on the relationship between chloroplast degradation and autophagy, a self-eating process conserved in all eukaryotic cells (Liu and Bassham, 2012). The accelerated leaf senescence observed in most autophagy-related (ATG) mutants suggests that autophagy might function as a strategy for carbon and nitrogen remobilization to the sink tissues by facilitating chloroplast degradation in the source tissues.

Three types of autophagy have been defined so far, including chaperone-mediated autophagy, macroautophagy and microautophagy (Mizushima and Komatsu, 2011). Chaperone-mediated autophagy, which depends on chaperone HSC70 and co-chaperones, has been reported in mamalian cells but not in yeast and plants (Mizushima and Komatsu, 2011). Macroautophagy occurs with the formation of a unique double membrane structure termed an autophagosome for the delivery of the cargos into the lysosomes/vacuole, and utilizes molecular machinery termed 
as ATG genes to generate the autophagosome. During autophagosome formation, an isolation membrane, named phagophore, engulfs and encloses the cargos to become a double membrane structure (Liu and Bassham, 2012). By contrast, during microautophagy, cargos are directly evaginated into the vacuole lumen by the vacuole membrane. Of note, microautophagy can be either ATG-dependent or ATGindependent (Mijaljica et al., 2011; Oku et al., 2017). These different types of autophagy have been implicated in cargo selectivity to facilitate the bulk or specific degradation of the target cargos under different conditions. In plant cells, excellent reviews have implicated that both macroautophagy and microautophagy pathways contribute to chloroplast degradation, and exhibit cargo specificity under different types of conditions (e.g., leaf senescence, carbon starvation or high light stress) by forming various types of structures (Xie et al., 2015; Izumi and Nakamura, 2018; Nakamura and Izumi, 2018; Otegui, 2018; Soto-Burgos et al., 2018). Here, we aim to compare these different pathways for the selective degradation of chloroplasts (here termed as chlorophagy), with an emphasis on the possible chloroplast receptors and related signals in this process.

\section{MACROAUTOPHAGY-LIKE DEGRADATION OF CHLOROPLASTS}

A macroautophagy-like process for either partial or whole chloroplast degradation utilizes the formation of autophagosomal structures and requires ATG proteins (Wada et al., 2009). Among these ATG proteins, ATG8 is widely used as an autophagosomal marker to label the autophagosomal structures (Yoshimoto et al., 2004; Contento et al., 2005; Zhuang et al., 2013, 2017, 2018; Spitzer et al., 2015). So far, several types of macroautophagyrelated structures have been reported, including the Rubiscocontaining body (RCB) (Ishida et al., 2008), the ATI1GFP Labels Plastid-Associated Body (ATI-PS body) (Michaeli et al., 2014), and small starch granule-like structures (SSTG) (Wang Y. et al., 2013; Figure 1).

\section{RCB Pathway}

The first observation of a macroautophagy-like process for chloroplast degradation came from the detection of Rubisco in small spherical bodies (around $1 \mu \mathrm{m}$ ) in both the cytoplasm and vacuole in wheat leaves, subsequently referred as RCBs (Chiba et al., 2003). Autophagosome-like double membranes are detected around these bodies (Figure 1). Another study using live-cell imaging to track the fate of RCBs (indicated by GFPlabeled Rubisco) in Arabidopsis and rice leaves demonstrated that the production of RCBs requires ATG genes including ATG5 or ATG7 (Ishida et al., 2008). Moreover, RCB fluorescent signals co-localize with the autophagosomal marker GFP-ATG8. This provides direct evidence that chloroplast proteins are degraded via autophagy by forming the RCBs and subsequent sequestration into autophagosomal structures. In addition, RCBs are highly induced by carbon deprivation and darkness, suggesting a role for autophagy in leaf carbon homeostasis by the degradation of chloroplast proteins via RCBs. In support of this, it has been observed that RCB numbers are increased in starchless mutants (Izumi et al., 2010, 2013). Therefore, RCBs represent a typical type of autophagic structure for chloroplast turnover.

It appears that the formation of RCBs is closely related to the extending stromule (Figure 1). Intriguingly, in the autophagy defective mutant atg5, many stromules are labeled by stromatargeted GFP, suggesting that RCBs are released from the extending stromules (Ishida et al., 2008). In addition, stromules are highly induced by different abiotic and biotic signals, such as exposure to ROS or high sucrose/glucose conditions (Brunkard et al., 2015; Hanson and Hines, 2018). Such plasticity in chloroplasts by forming extending stromules in response to diverse stimuli might provide an efficient way for the removal of chloroplast materials. By GFP labeling in the confocal microscope and immunolocalization in transmission electron microscope (TEM), it was found that only Rubisco, but not chlorophyll, is detected in the stromules (Kwok and Hanson, 2004; Holzinger et al., 2008). This explains why thylakoid membranes are not detected in the RCBs. Intriguingly, extended stromules were also observed in a mutant defective in the Endosomal sorting complex required for transport (ESCRT) protein, CHMP1 (Spitzer et al., 2015). A primary role for the ESCRT complex in multivesicular body (MVB) biogenesis and endosomal sorting has been well characterized in plant cells (Gao et al., 2017). It is therefore proposed that CHMP1 might play an additional role in autophagosome maturation, thus a malfunction of CHMP1 might lead to an incomplete autophagosome, and the delay of RCB sequestration, resulting in the accumulation of phagophorelike structures and cytoplasmic RCBs (Spitzer et al., 2015).

\section{ATI-PS Body Pathway}

Another type of autophagy-related degradation structure with a size around $1 \mu \mathrm{m}$, is termed the ATI-PS body, which is mediated by the ATI proteins (Michaeli et al., 2014). ATI proteins are plant-specific and no counterparts have been identified in non-plant species (Honig et al., 2012). They directly interact with ATG8 via the ATG8-interacting motif (AIM) and were previously identified as being distributed on the endoplasmic reticulum (ER). However, upon sugar starvation, ATI is detected on the outer membrane of chloroplast but not on stromules, precluding a similar origin to RCBs (Michaeli et al., 2014). Another major difference of ATI-PS bodies is that they contain different chloroplast cargos, including stromal, thylakoid and envelope proteins. Although the ATI-PS bodies are suggested to be derived from the chloroplast thylakoid, it raises a question as to how ATI, which is predicted to be a single transmembrane protein on the ER, is relocalized into the chloroplast lumen from the chloroplast outer membrane. Of note, the release of ATI-PS body from plastid is ATG-independent, but its delivery into the vacuole requires the ATG machinery (Michaeli et al., 2014).

\section{SSTG Pathway}

Starch granules, which are wildly deposited in the chloroplast, serve as an essential carbon reservoir by converting starch into sugar (Malinova et al., 2018). A previous study revealed that starch contents are greatly decreased (about 70\%) in atg $4 a 4 b-1$ upon exposure to the darkness (Izumi et al., 2010). In another 
study, electron microscopy has shown that a small starch granulelike structure (usually with a diameter of $<0.5 \mu \mathrm{m}$ ) was captured in the cytosol and sequestered into the autophagic bodies (Wang Y. et al., 2013). These SSGLs were clearly labeled by YFP-tagged granule-bound starch synthase I (GBSSI-YFP), a starch granule marker, and also colocalized with the autophagosome marker CFP-ATG8f. It also appears that the occurrence of stromules may contribute to the release of SSGL, which is supported by the detection of SSGL in the stromules by both confocal and electron microscopy (Wang Y. et al., 2013). Similar to RCBs and ATI-PS bodies, the number of vacuole-localized SSGLs was greatly reduced after blocking autophagic activity via ATG6 silencing, suggesting that the degradation of SSGLs are also ATG-dependent (Wang Y. et al., 2013).

\section{Whole Chloroplast Pathway}

Previous study has shown that whole chloroplasts are delivered to vacuoles in individually darkened leaves which display accelerated senescence due to sugar starvation (Wada et al., 2009). A recent study has also showed that whole chloroplasts can be targeted for degradation by autophagy upon exposure to UV light (Izumi et al., 2017). After UV light exposure, the autophagosomal membrane, as labeled by GFP-ATG8a, captures the whole chloroplast and encloses it into a completed autophagosome. These autophagosomal structures are much larger than the previously described chlorophagy-related structures, and can be readily detected in the vacuole as well. Moreover, plants lacking autophagic activity have less vacuolar delivery of these UV light-triggered structures into the vacuole and display a higher sensitivity to UV-B exposure. It is suggested that the invagination of the entire chloroplast is different from the RCB pathway, as it occurs independent of the activation of the RCB pathway. However, in both $\operatorname{atg} 5$ and $\operatorname{atg} 7$ mutants, damaged chloroplasts with extended stromules also accumulated upon UV-B exposure (Izumi et al., 2017), raising the possibility that stromule formation might contribute to whole chloroplast degradation as well.

\section{MICROAUTOPHAGY-LIKE DEGRADATION OF CHLOROPLASTS}

In comparison to macroautophagy, microautophagy mediates the degradation of chloroplast by direct invagination of the chloroplast contents via the vacuole membrane (Figure 1). A recent study showed that high-intensity light (HL) will trigger chloroplast envelope damage and lead to chloroplast swelling (Nakamura et al., 2018). In addition, overexpression of VESICLE INDUCING PROTEIN IN PLASTID1 (VIPP1), a protein that regulates chloroplast envelope integrity, causes the formation of abnormal swollen chloroplasts (Nakamura et al., 2018). These

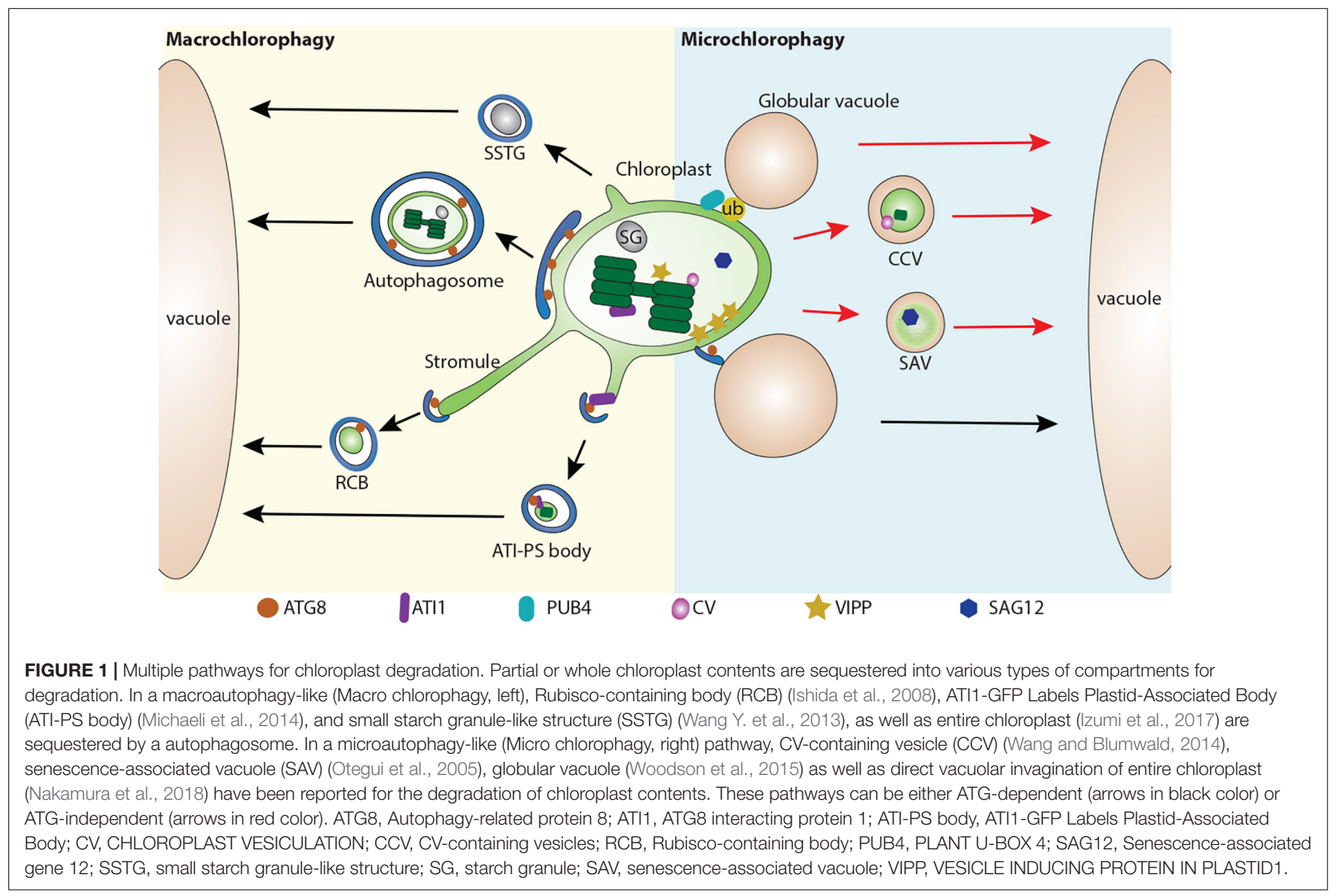


swollen chloroplasts are detected in the vacuole under $\mathrm{HL}$ or VIPP1 overexpression conditions. Interestingly, it appears that the swollen chloroplasts are initially recognized by the ATG8-containing structures prior to vacuole invagination. Furthermore, confocal imaging analysis showed that the entire swollen chloroplast is directly engulfed by the GFP- $\delta$ TIP labeled tonoplast into the vacuole, while this process is absent in the $\operatorname{atg} 5$ mutant, supporting the involvement of an ATGdependent microautophagy-like process. The role of the ATG8labeled structure is suggested to serve as a selective platform for chloroplast recognition via an interaction between the chlorophagy receptor(s) and ATG8, in a manner similar to that

TABLE 1 | Predicted chloroplast outer membrane proteins containing the ATG8-interacting motif in Arabidopsis.

\begin{tabular}{|c|c|c|c|c|}
\hline Name & Gene & $\begin{array}{l}\text { ATG8- } \\
\text { interacting } \\
\text { motif }\end{array}$ & $\begin{array}{l}\text { Position from } \\
\text { (aa) }\end{array}$ & $\begin{array}{l}\text { Position to } \\
\text { (aa) }\end{array}$ \\
\hline Toc33 & At1g02280 & EFFGKL & 24 & 29 \\
\hline \multirow[t]{2}{*}{ Toc34 } & At5g05000 & REWIGI & 8 & 13 \\
\hline & & NLFNKI & 237 & 242 \\
\hline Toc64/OEP64 & At3g17970 & NLWVLL & 7 & 12 \\
\hline Toc75l & At1g35860 & YSFANV & 55 & 60 \\
\hline Toc75III & At3g4674 & GMFEKV & 223 & 228 \\
\hline Toc75-IV & At4g09080 & / & / & / \\
\hline Toc75-V/OEP80 & At5g19620 & / & / & / \\
\hline \multirow[t]{4}{*}{ Toc159 } & At4g02510 & GEFEPV & 286 & 291 \\
\hline & & KTYASV & 23 & 28 \\
\hline & & YRYRYL & 1265 & 1270 \\
\hline & & SIYKSI & 1510 & 1515 \\
\hline OEP7 & At3g52420 & LGWLAI & 19 & 24 \\
\hline OEP9 & At1g16000 & / & / & / \\
\hline OEP61 & At5g21990 & ADFARI & 24 & 29 \\
\hline OEP21A & At1g20816 & EMFEKV & 138 & 143 \\
\hline OEP21B & At1g76405 & EMFDKV & 138 & 143 \\
\hline \multirow[t]{3}{*}{ OEP24A } & At1g45170 & PSFNGL & 43 & 48 \\
\hline & & PGFFII & 55 & 60 \\
\hline & & LKYTY & 126 & 131 \\
\hline OEP24B & At5g42960 & GSFI V & 57 & 62 \\
\hline OEP37 & At2g43950 & LGWASL & 298 & 303 \\
\hline PDV1 & At5g53280 & PGYVFI & 62 & 67 \\
\hline PDV2 & At2g16070 & KDFEVL & 130 & 135 \\
\hline \multirow{2}{*}{$\begin{array}{l}\text { Cytochrome } \\
\text { b5 }\end{array}$} & At1g26340 & DCWWI & 21 & 26 \\
\hline & & KQYWW & 112 & 117 \\
\hline / & At4g16070 & DSWTGI & 412 & 417 \\
\hline / & At4g27610 & PNWILI & 22 & 27 \\
\hline \multirow[t]{4}{*}{ / } & At5g11250 & FSYDAL & 481 & 486 \\
\hline & & IGFFTL & 14 & 19 \\
\hline & & RDFDGL & 234 & 239 \\
\hline & & IYSGL & 1162 & 1167 \\
\hline \multirow[t]{2}{*}{ TGD4 } & AT3g06960 & PSFSPI & 64 & 69 \\
\hline & & AWWPGL & 193 & 198 \\
\hline
\end{tabular}

* The order of the predicted motifs are based on the score obtained on the iLIR web tool (http://repeat.biol.ucy.ac.cy/iLIR/). aa, amino acid. for the ATI-PS body. Alternatively, the formation of ATG8-sac structures may facilitate the deposition of the cap-like structure at the chloroplast to control the docking and fusion between the chloroplast membrane and the tonoplast, followed by the release of the chloroplast contents into the vacuole lumen.

Other studies have also reported other types of structures for the execution of chloroplast degradation in a microautophagylike manner, although they were not initially defined as a microautophagy-like process. For example, senescenceassociated vacuoles (SAVs), which are characterized by a senescence-induced cysteine protease Senescence-associated gene 12 (SAG12), were identified as a distinct type of lytic compartment during leaf senescence (Otegui et al., 2005). SAVs display similar characteristics to the lytic vacuole as they are stained by LysoTracker red or neutral red, although they lack the tonoplast marker $\gamma$-TIP (Otegui et al., 2005). Another study showed that isolated SAVs contain stromal proteins including Rubisco and glutamine synthetase, but lack thylakoid proteins (Martinez et al., 2008). It was claimed that SAVs are still formed in the atg7 mutant, thus representing a separate pathway for chloroplast turnover (Otegui et al., 2005). However, in another study, by targeting chloroplasts with CHLOROPLAST VESICULATION (CV), it is shown that $\mathrm{CV}$-containing vesicles (CCVs) are accumulated under abiotic stress and downregulated by cytokinin (Wang and Blumwald, 2014). Chloroplast materials including stroma, envelope, and thylakoid proteins are identified in these CCVs. The authors also showed that the formation of CCVs is autophagy-independent and is separate from the autophagosome marker GFP-ATG8a. Instead, bimolecular fluorescence complementation (BiFC) assays showed that $\mathrm{CV}$ interact with $\mathrm{PsbO}$ protein, a component of photosystem II complex on the thylakoid membrane, suggesting that CCVs may arise from within the chloroplast. Recently, it has also been shown that $\mathrm{CV}$ is regulated by a NAC transcription factor RD26 (Kamranfar et al., 2018), which also regulates ABA-related genes (Zhang and Gan, 2012), implying a feedback regulation between ABA signaling and chloroplast degradation via the CCVs. However, lacking an evident observation of the intermediate structures for the formation of SAVs and the CCVs renders their origins obscure.

\section{CHLOROPHAGY-RELATED MEMBRANE RECEPTORS IN PLANTS}

A critical question in regarding to chlorophagy is how selectivity for chloroplast contents is executed during this process. Specific autophagic receptors are known to function in different types of autophagy, such as mitophagy, pexophagy and ER-phagy (Zaffagnini and Martens, 2016). The organelle components are recognized by autophagic receptors for docking to the autophagosomal structure or the vacuole membrane. So far, the autophagic receptors identified usually contain a canonical AIM, and a specific cargo interacting domain, and are either ubiquitin-dependent or ubiquitin-independent (Zaffagnini and Martens, 2016). 
However, with respect to the chloroplast being specific for plant cells, it is not surprising that plant-unique receptors might be involved in chlorophagy. ATI1/2 represents a type of plant-specific receptor for chlorophagy which interacts with ATG8 upon sugar starvation (Michaeli et al., 2014). Several chloroplast-localized proteins have also been found to interact with ATI by the split ubiquitin yeast two-hybrid assay, and it was suggested that these proteins are sequestered into the ATI-PS body via ATI interaction (Michaeli et al., 2014). However, ATI is localized on the ER under normal conditions and how the sugar signal activates the recruitment of ATI onto the outer membrane of the chloroplast remains unknown (Honig et al., 2012). An extensive supply of lipid precursors from the endoplasmic reticulum (ER) to the chloroplast have been implicated to be mediated by a group of TRIGALACTOSYLDIACYLGLYCEROL (TGD) proteins, and only TGD4 has been shown to be distributed on the outer membrane of the chloroplast (Fan et al., 2015). One possibility would be that ATI is translocated onto the chloroplast via ER-chloroplast contact sites, so that it can be mobilized via lipid exchange to aid the initiation of the ATI-PS body (Zhuang et al., 2016).

In addition to ATI1/2, there are no proteins on the plant chloroplast surface that have been experimentally tested to have a similar function during chlorophagy. Using a Bioinformatic tool for prediction of proteins that may interact with ATG8 (Kalvari et al., 2014), and based on the available experimental information of the chloroplast outer membrane proteins in Arabidopsis (Lee et al., 2014), a set of predicted AIM-containing proteins in Arabidopsis is listed in Table 1, including TOC159, TOC75 and TOC33. TOC159, TOC75, and TOC33 are inserted into the outer membrane of chloroplast, forming the chloroplast protein TOC import complex for the import of chloroplast proteins (Lee et al., 2014). Particularly, post-modifications of the TOC complex under various induction conditions have been reported (Agne et al., 2010; Ling et al., 2012; Wang P. et al., 2013; Woodson et al., 2015; Shanmugabalaji et al., 2018; Figure 2). In addition to the TOC complex, potential AIMs were also identified in the outer envelope protein (OEP) complex, which serves as an alternative pathway for chloroplast protein import (Lee et al., 2014). OEP7 and OEP9 have been shown to function together with heat shock protein Hsp17.8 and AKR2A cofactors in targeting membrane proteins to plastid outer membranes under normal physiological conditions (Niehaus et al., 2014; Kim et al., 2015). To enable the efficient recognition by the autophagosomal or vacuolar membrane, it is possible that these chloroplast outer membrane proteins might serve as receptors by binding to the ATG or non-ATG chlorophagy regulators. However, since these plant-specific chloroplast proteins lack counterparts in either yeast or mammalian cells, more effort

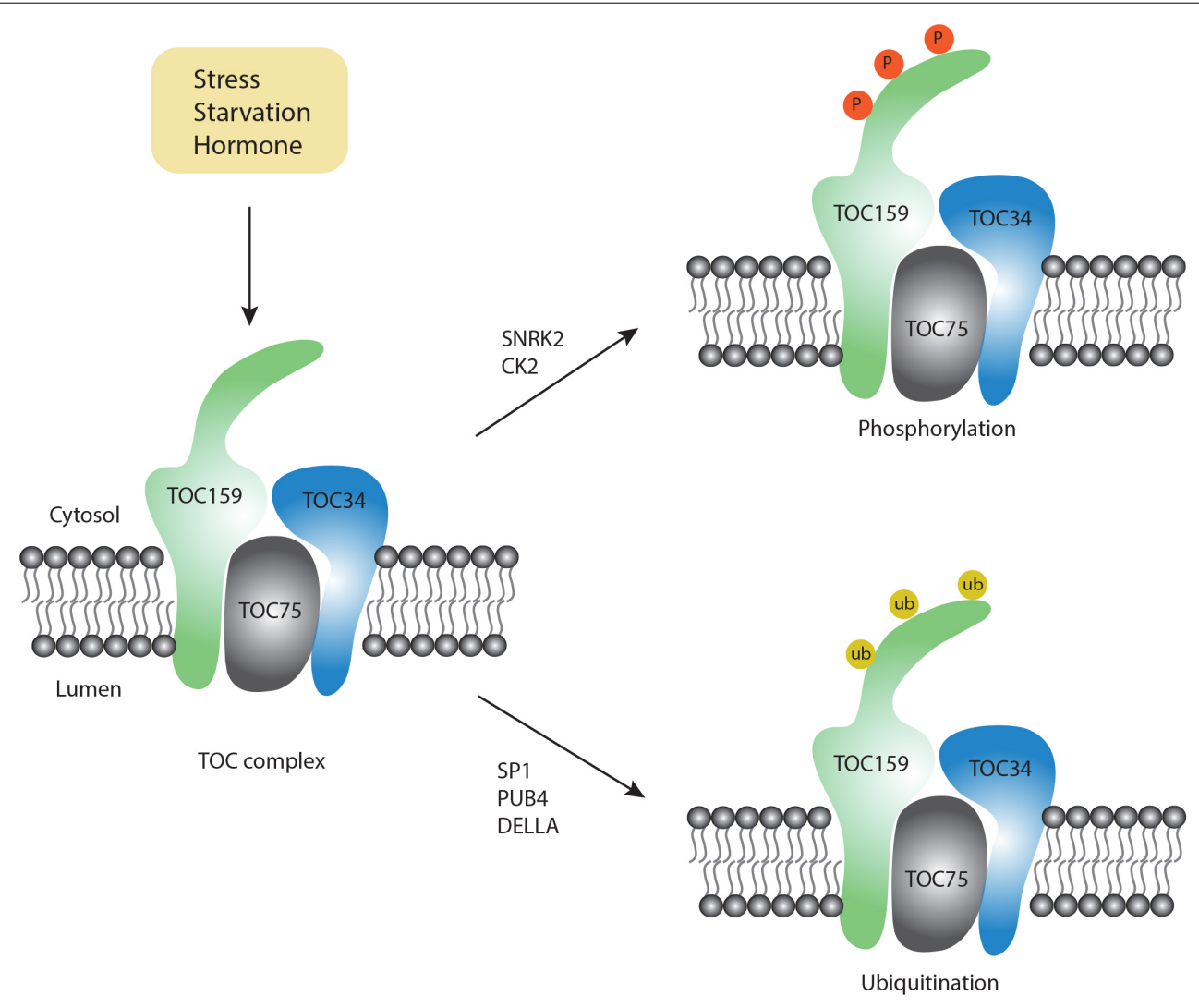

FIGURE 2 | Post-modification on the TOC complex. Upon exposure to different conditions, two types of modifications occur on the TOC complex, including phosphorylation (orange color) and ubiquitination (yellow color). 
will be required in the future to validate the predicted AIMs and their possible roles in different types of chloroplast degradation pathways.

\section{POSSIBLE SIGNALS AND POST-TRANSLATIONAL MODIFICATION FOR THE CHLOROPLAST PROTEINS DURING CHLOROPHAGY}

Although chlorophagy is highly induced by various stress conditions, the molecular signals for chlorophagy activation remain unknown. Notably, RCB formation requires stromule formation, which is highly inducible under starvation and stress conditions (Ishida et al., 2008). For example, it has been reported that stromule number is increased upon ABA treatment (Gray et al., 2012). On the other hand, ABA modulates the activity of downstream factors like ABI and P2CC, which further activate the SnRK2 kinase complex (Soon et al., 2012). Particularly, the SnRK complex also serves as a critical upstream regulator for autophagic activation by phosphorylation of a set of ATG proteins, e.g., ATG1 (Soto-Burgos et al., 2018). Although there is no direct evidence to link ABA signaling with autophagy, a recent study using quantitative phosphoproteomics showed that phosphorylation of TOC159 homologs TOC132 and TOC120 occurs in an ABA-dependent manner only in the SnRK2-deficient mutant background (Wang P. et al., 2013). Furthermore, in vitro phosphorylation assays have demonstrated that TOC159 might be directly phosphorylated by recombinant SnRK2 proteins (Wang P. et al., 2013). In another study, phosphorylation of recombinant A-domain of TOC159 was also observed when incubated with casein kinase 2 (CK2)like proteins (Agne et al., 2010), an evolutionarily conserved serine/threonine protein kinase to function in circadian clock regulation. Recently, it was shown that CK2 also participates in the ABA-activated SnRK2 signaling pathway (Vilela et al., 2015). Therefore, it is possible that SnRK2- or CK2- dependent phosphorylation of chloroplast membrane proteins or the ATG proteins activates chlorophagy in coordination with the ABA signaling pathway. It will be interesting to test whether the phosphorylation of these chloroplast membrane-associated proteins would serve as a signal to recruit the ATG machinery onto the chloroplast membrane.

In addition to phosphorylation, another type of essential post-translational modification, ubiquitination, might provide the specificity for the selection of chloroplast cargos. Emerging evidence from both yeast and mammalian cells supports a close interplay between ubiquitination and autophagy (Shaid et al., 2013). In plants, it is well known that ubiquitination plays a critical role in chloroplast biogenesis during plastid developmental transitions (e.g., proplastid to chloroplast in seed germination). In a recent study, it was reported that during germination, the DELLA proteins promote TOC159 degradation via ubiquitination to modulate proplastid to chloroplast transition during early plant development (Shanmugabalaji et al., 2018). Although it is claimed that ubiquitinated
TOC159 is degraded via the proteasome pathway, whether ubiquitinated TOC159 may be targeted by chlorophagy awaits to be determined. Of note, TOC159 has previously been identified as a substrate of another E3 ligase SP1, which is distributed on the outer membrane of the chloroplast to regulate chloroplast biogenesis (Ling et al., 2012). Interestingly, MUL1, the closest homolog of SP1 in humans, has been shown to regulate the ULK1 (ATG1 homolog in human) activity and mitophagy negatively (Li et al., 2015; Rojansky et al., 2016). Upon selenite treatment, it was observed that ULK1 translocates to mitochondria to interact with MUL1. In plants, SP1 has been detected on peroxisomes, mitochondria as well as chloroplasts (Pan and Hu, 2018). Additionally, it was reported that Parkin, a E3 ubiquitin ligase in mammalian cells, interacts with the mitochondrial outer membrane protein VDAC to regulate its ubiquitination for the targeting of mitochondria during mitophagy (Geisler et al., 2010). Hence, it is possible that SP1 might play an additional role during chlorophagy and future work is needed to identify potential SP1 substrates during chlorophagy.

Recently, in a screening for a suppressor of $f c 2$-triggered chloroplast degradation, a E3 ligase PLANT U-BOX 4 (PUB4) was identified (Woodson et al., 2015). It was implied that PUB4 functions in chloroplast turnover via ubiquitination of the chloroplast proteins during dark-to-light transitions against ROS. Of note, loss-of-function of the chloroplast protein import machinery (TOC33 and TOC159), also suppresses $f c 2$-triggered chloroplast degradation, implying a coordination between the chloroplast import machinery and degradation. It appears that a pub4 mutant exhibits less sensitivity to carbon starvation than the atg mutant, thus the authors claimed that the PUB4-mediated chloroplast degradation is likely to be independent of autophagy. However, during the PUB4-dependent chloroplast degradation process, it was observed that the damaged chloroplast directly fuses with a globular vacuole, which is quite similar to the microautophagy pathway through direct vacuolar invagination. It should be pointed out that the ATG machinery is dispensable during microautophagy and diverse molecular machineries are identified in other species. For instance, the ESCRT machinery but not the ATG machinery has been demonstrated to participate in the incorporation of cytoplasmic proteins into the vacuole (Oku et al., 2017).

\section{PERSPECTIVE}

Chloroplast homeostasis is critical for efficient nutrient recycling and remobilization. A significant role for autophagy in senescent leaves is to avoid the accumulation of toxic products from the chloroplast by removing the damaged or excessive chloroplast contents. For instance, chloroplasts produce ROS and stress hormones (e.g., salicylic acid and ABA precursor), both of which can alter nuclear gene expression and accelerate leaf senescence (Yoshimoto et al., 2009; Schippers et al., 2015). Thus, degradation of chloroplasts by autophagy may promote cell survival, and contribute to the natural turnover of aging chloroplasts to overcome early leaf senescence and cell death. However, so 
far, little is known about how chlorophagy is regulated and how the selectivity of chloroplast materials is achieved. We anticipate that more efforts will be put forward in future toward the identification of novel chloroplast regulators to link with the ATG machinery. In particular, identification of the distinct chlorophagy receptors for different pathways as well as their interaction network should provide more insights into how these different pathways are coordinated for chloroplast turnover. In addition, although previous studies visualizing chlorophagyrelated structures have mainly relied on $2 \mathrm{D}$ transmission electron microscopy images or confocal microscopy imaging, details on the intermediate structures are still missing. Outstanding questions are as follows: Why are there so many types of pathways/structures and are they related? What happens if a pathway/structure is inhibited? How are vesicles initiated within the double-membrane chloroplast (e.g., ATI-PS)? How does the outer membrane of chloroplast fuse with the vacuole? Given the complexity of chloroplast morphology, a combination of advanced techniques such as 3D electron microscopy and dynamic imaging should provide more insights into chlorophagy at the cellular level in the future.

\section{REFERENCES}

Agne, B., Andres, C., Montandon, C., Christ, B., Ertan, A., Jung, F., et al. (2010). The acidic A-domain of Arabidopsis Toc159 occurs as a hyperphosphorylated protein. Plant Physiol. 153, 1016-1030. doi: 10.1104/pp.110. 158048

Brunkard, J. O., Runkel, A. M., and Zambryski, P. C. (2015). Chloroplasts extend stromules independently and in response to internal redox signals. Proc. Natl. Acad. Sci. U.S.A. 112, 10044-10049. doi: 10.1073/pnas.1511570112

Chiba, A., Ishida, H., Nishizawa, N. K., Makino, A., and Mae, T. (2003). Exclusion of ribulose-1,5-bisphosphate carboxylase/oxygenase from chloroplasts by specific bodies in naturally senescing leaves of wheat. Plant Cell Physiol. 44, 914-921. doi: 10.1093/pcp/pcg118

Contento, A. L., Xiong, Y., and Bassham, D. C. (2005). Visualization of autophagy in Arabidopsis using the fluorescent dye monodansylcadaverine and a GFPAtATG8e fusion protein. Plant J. 42, 598-608. doi: 10.1111/j.1365-313X.2005. 02396.x

Fan, J. L., Zhai, Z. Y., Yan, C. S., and Xu, C. C. (2015). Arabidopsis TRIGALACTOSYLDIACYLGLYCEROL5 interacts with TGD1, TGD2, and TGD4 to facilitate lipid transfer from the endoplasmic reticulum to plastids. Plant Cell 27, 2941-2955. doi: 10.1105/tpc.15.00394

Gao, C. J., Zhuang, X. H., Shen, J. B., and Jiang, L. W. (2017). Plant ESCRT complexes: moving beyond endosomal sorting. Trends Plant Sci. 22, 986-998. doi: 10.1016/j.tplants.2017.08.003

Geisler, S., Holmstrom, K. M., Skujat, D., Fiesel, F. C., Rothfuss, O. C., Kahle, P. J., et al. (2010). PINK1/Parkin-mediated mitophagy is dependent on VDAC1 and p62/SQSTM1. Nat. Cell Biol. 12, 119-131. doi: 10.1038/ncb2012

Gray, J. C., Hansen, M. R., Shaw, D. J., Graham, K., Dale, R., Smallman, P., et al. (2012). Plastid stromules are induced by stress treatments acting through abscisic acid. Plant J. 69, 387-398. doi: 10.1111/j.1365-313X.2011. 04800.x

Hanson, M. R., and Hines, K. M. (2018). Stromules: probing formation and function. Plant Physiol. 176, 128-137. doi: 10.1104/pp.17.01287

Holzinger, A., Kwok, E. Y., and Hanson, M. R. (2008). Effects of arc3, arc5 and arc6 mutations on plastid morphology and stromule formation in green and nongreen tissues of Arabidopsis thaliana. Photochem. Photobiol, 84, 1324-1335. doi: 10.1111/j.1751-1097.2008.00437.x

Honig, A., Avin-Wittenberg, T., Ufaz, S., and Galili, G. (2012). A new type of compartment, defined by plant-specific Atg8-interacting proteins, is induced upon exposure of Arabidopsis plants to carbon starvation. Plant Cell 24, 288303. doi: 10.1105/tpc.111.093112

\section{AUTHOR CONTRIBUTIONS}

$\mathrm{XZ}$ designed the concept and the organization of the manuscript. $\mathrm{XZ}$ and LJ wrote and edited the manuscript.

\section{FUNDING}

This work was supported by Grants from the National Natural Science Foundation of China (31670179 and 91854201), the Research Grants Council of Hong Kong (G-CUHK403/17, G-CUHK404/18, CUHK14130716, CUHK14102417, 14100818, C4011-14R, C4012-16E, C4002-17G, R4005-18F, and AoE/M05/12) and CUHK Research Committee.

\section{ACKNOWLEDGMENTS}

We apologize to researchers whose work has not been included in this manuscript owing to space limit.

Ishida, H., Yoshimoto, K., Izumi, M., Reisen, D., Yano, Y., Makino, A., et al. (2008). Mobilization of rubisco and stroma-localized fluorescent proteins of chloroplasts to the vacuole by an ATG gene-dependent autophagic process. Plant Physiol. 148, 142-155. doi: 10.1104/pp.108.122770

Izumi, M., Hidema, J., Makino, A., and Ishida, H. (2013). Autophagy contributes to nighttime energy availability for growth in Arabidopsis. Plant Physiol. 161, 1682-1693. doi: 10.1104/pp.113.215632

Izumi, M., Ishida, H., Nakamura, S., and Hidema, J. (2017). Entire photodamaged chloroplasts are transported to the central vacuole by autophagy. Plant Cell 29, 377-394. doi: 10.1105/tpc.16.00637

Izumi, M., and Nakamura, S. (2018). Chloroplast protein turnover: the influence of extraplastidic processes, including autophagy. Int. J. Mol. Sci. 19:E828. doi: 10.3390/ijms 19030828

Izumi, M., Wada, S., Makino, A., and Ishida, H. (2010). The autophagic degradation of chloroplasts via Rubisco-containing bodies is specifically linked to leaf carbon status but not nitrogen status in Arabidopsis. Plant Physiol. 154, 1196-1209. doi: 10.1104/pp.110.158519

Jarvis, P., and Lopez-Juez, E. (2014). Biogenesis and homeostasis of chloroplasts and other plastids (vol 14, pg 787, 2013). Nat. Rev. Mol. Cell Biol. 15:147. doi: $10.1038 / \mathrm{nrm} 3702$

Kalvari, I., Tsompanis, S., Mulakkal, N. C., Osgood, R., Johansen, T., Nezis, I. P., et al. (2014). iLIR A web resource for prediction of Atg8-family interacting proteins. Autophagy 10, 913-925. doi: 10.4161/auto.28260

Kamranfar, I., Xue, G. P., Tohge, T., Sedaghatmehr, M., Fernie, A. R., Balazadeh, S., et al. (2018). Transcription factor RD26 is a key regulator of metabolic reprogramming during dark-induced senescence. New Phytol. 218, 1543-1557. doi: 10.1111/nph.15127

Kim, D. H., Lee, J. E., Xu, Z. Y., Geem, K. R., Kwon, Y., Park, J. W., et al. (2015). Cytosolic targeting factor AKR2A captures chloroplast outer membranelocalized client proteins at the ribosome during translation. Nat. Commun. 6:6843. doi: 10.1038/ncomms7843

Kwok, E. Y., and Hanson, M. R. (2004). GFP-labelled Rubisco and aspartate aminotransferase are present in plastid stromules and traffic between plastids. J. Exp. Bot. 55, 595-604. doi: 10.1093/jxb/erh062

Lee, J., Kim, D. H., and Hwang, I. (2014). Specific targeting of proteins to outer envelope membranes of endosymbiotic organelles, chloroplasts, and mitochondria. Front. Plant Sci. 5:173. doi: 10.3389/fpls.2014.00173

Li, J., Qi, W., Chen, G., Feng, D., Liu, J. H., Ma, B., et al. (2015). Mitochondrial outer-membrane E3 ligase MUL1 ubiquitinates ULK1 and regulates seleniteinduced mitophagy. Autophagy 11, 1216-1229. doi: 10.1080/15548627.2015. 1017180 
Ling, Q. H., Huang, W. H., Baldwin, A., and Jarvis, P. (2012). Chloroplast biogenesis is regulated by direct action of the ubiquitin-proteasome system. Science 338, 655-659. doi: 10.1126/science. 1225053

Liu, Y., and Bassham, D. C. (2012). Autophagy: pathways for self-eating in plant cells. Annu. Rev. Plant Biol. 63, 215-237. doi: 10.1146/annurev-arplant-042811105441

Malinova, I., Qasim, H. M., Brust, H., and Fettke, J. (2018). Parameters of starch granule genesis in chloroplasts of Arabidopsis thaliana. Front. Plant Sci. 9:761. doi: $10.3389 /$ fpls.2018.00761

Martinez, D. E., Costa, M. L., Gomez, F. M., Otegui, M. S., and Guiamet, J. J. (2008). 'Senescence-associated vacuoles' are involved in the degradation of chloroplast proteins in tobacco leaves. Plant J. 56, 196-206. doi: 10.1111/j.1365-313X.2008. 03585.x

Michaeli, S., Honig, A., Levanony, H., Peled-Zehavi, H., and Galili, G. (2014). Arabidopsis ATG8-INTERACTING PROTEIN1 is involved in autophagydependent vesicular trafficking of plastid proteins to the vacuole. Plant Cell 26, 4084-4101. doi: 10.1105/tpc.114.129999

Mijaljica, D., Prescott, M., and Devenish, R. J. (2011). Microautophagy in mammalian cells revisiting a 40-year-old conundrum. Autophagy 7, 673-682. doi: 10.4161/auto.7.7.14733

Mizushima, N., and Komatsu, M. (2011). Autophagy: renovation of cells and tissues. Cell 147, 728-741. doi: 10.1016/j.cell.2011.10.026

Nakamura, S., Hidema, J., Sakamoto, W., Ishida, H., and Izumi, M. (2018). Selective elimination of membrane-damaged chloroplasts via microautophagy. Plant Physiol. 177, 1007-1026. doi: 10.1104/pp.18.00444

Nakamura, S., and Izumi, M. (2018). Regulation of chlorophagy during photoinhibition and senescence: lessons from mitophagy. Plant Cell Physiol. 59, 1135-1143. doi: 10.1093/pcp/pcy096

Niehaus, T. D., Richardson, L. G., Gidda, S. K., Elbadawi-Sidhu, M., Meissen, J. K., Mullen, R. T., et al. (2014). Plants utilize a highly conserved system for repair of NADH and NADPH hydrates. Plant Physiol. 165, 52-61. doi: 10.1104/pp.114. 236539

Oku, M., Maeda, Y., Kagohashi, Y., Kondo, T., Yamada, M., Fujimoto, T., et al. (2017). Evidence for ESCRT- and clathrin-dependent microautophagy. J. Cell Biol. 216, 3263-3274. doi: 10.1083/jcb.201611029

Otegui, M. S. (2018). Vacuolar degradation of chloroplast components: autophagy and beyond. J. Exp. Bot. 69, 741-750. doi: 10.1093/jxb/erx234

Otegui, M. S., Noh, Y. S., Martinez, D. E., Vila Petroff, M. G., Andrew Staehelin, L., Amasino, R. M., et al. (2005). Senescence-associated vacuoles with intense proteolytic activity develop in leaves of Arabidopsis and soybean. Plant J. 41, 831-844. doi: 10.1111/j.1365-313X.2005.02346.x

Pan, R. H., and Hu, J. P. (2018). The Arabidopsis E3 ubiquitin ligase SP1 targets to chloroplasts, peroxisomes, and mitochondria. Plant Physiol. 176, 480-482. doi: 10.1104/pp.17.01536

Rojansky, R., Cha, M. Y., and Chan, D. C. (2016). Elimination of paternal mitochondria in mouse embryos occurs through autophagic degradation dependent on PARKIN and MUL1. elife 5:e17896. doi: 10.7554/eLife.17896

Schippers, J. H. M., Schmidt, R., Wagstaff, C., and Jing, H. C. (2015). Living to die and dying to live: the survival strategy behind leaf senescence. Plant Physiol. 169, 914-930. doi: 10.1104/pp.15.00498

Shaid, S., Brandts, C. H., Serve, H., and Dikic, I. (2013). Ubiquitination and selective autophagy. Cell Death Differ. 20, 21-30. doi: 10.1038/cdd.2012.72

Shanmugabalaji, V., Chahtane, H., Accossato, S., Rahire, M., Gouzerh, G., LopezMolina, L., et al. (2018). Chloroplast biogenesis controlled by DELLA-TOC159 interaction in early plant development. Curr. Biol. 28, 2616-2623.e5 doi: 10. 1016/j.cub.2018.06.006

Siqueira, J. A., Hardoim, P., Ferreira, P. C. G., Nunes-Nesi, A., and Hemerly, A. S. (2018). Unraveling interfaces between energy metabolism and cell cycle in plants. Trends Plant Sci. 23, 731-747. doi: 10.1016/j.tplants.2018. 05.005

Soon, F. F., Ng, L. M., Zhou, X. E., West, G. M., Kovach, A., Tan, M. H. E., et al. (2012). Molecular mimicry regulates ABA signaling by SnRK2 kinases and PP2C phosphatases. Science 335, 85-88. doi: 10.1126/science. 1215106

Soto-Burgos, J., Zhuang, X. H., Jiang, L. W., and Bassham, D. C. (2018). Dynamics of autophagosome formation. Plant Physiol. 176, 219-229. doi: 10.1104/pp.17. 01236
Spitzer, C., Li, F. Q., Buono, R., Roschzttardtz, H., Chung, T. J., Zhang, M., et al. (2015). The endosomal protein CHARGED MULTIVESICULAR BODY PROTEIN1 regulates the autophagic turnover of plastids in Arabidopsis. Plant Cell 27, 391-402. doi: 10.1105/tpc.114.135939

Vilela, B., Najar, E., Lumbreras, V., Leung, J., and Pages, M. (2015). Casein kinase 2 negatively regulates abscisic acid-activated SnRK2s in the core abscisic acid-signaling module. Mol. Plant 8, 709-721. doi: 10.1016/j.molp.2014.12.012

Wada, S., Ishida, H., Izumi, M., Yoshimoto, K., Ohsumi, Y., Mae, T., et al. (2009). Autophagy plays a role in chloroplast degradation during senescence in individually darkened leaves. Plant Physiol. 149, 885-893. doi: 10.1104/pp. 108.130013

Wang, P., Xue, L., Batelli, G., Lee, S., Hou, Y. J., Van Oosten, M. J., et al. (2013). Quantitative phosphoproteomics identifies SnRK2 protein kinase substrates and reveals the effectors of abscisic acid action. Proc. Natl. Acad. Sci. U.S.A. 110, 11205-11210. doi: 10.1073/pnas.1308974110

Wang, S. H., and Blumwald, E. (2014). Stress-induced chloroplast degradation in Arabidopsis is regulated via a process independent of autophagy and senescence-associated vacuoles. Plant Cell 26, 4875-4888. doi: 10.1105/tpc.114. 133116

Wang, Y., Yu, B. J., Zhao, J. P., Guo, J. B., Li, Y., Han, S. J., et al. (2013). Autophagy contributes to leaf starch degradation. Plant Cell 25, 1383-1399. doi: $10.1105 /$ tpc.112.108993

Woodson, J. D., Joens, M. S., Sinson, A. B., Gilkerson, J., Salom, P. A., Weigel, D., et al. (2015). Ubiquitin facilitates a quality-control pathway that removes damaged chloroplasts. Science 350, 450-454. doi: 10.1126/science.aac7444

Xie, Q. J., Michaeli, S., Peled-Zehavi, N., and Galili, G. (2015). Chloroplast degradation: one organelle, multiple degradation pathways. Trends Plant Sci. 20, 264-265. doi: 10.1016/j.tplants.2015.03.013

Yoshimoto, K., Hanaoka, H., Sato, S., Kato, T., Tabata, S., Noda, T., et al. (2004). Processing of ATG8s, ubiquitin-like proteins, and their deconjugation by ATG4s are essential for plant autophagy. Plant Cell 16, 2967-2983. doi: $10.1105 /$ tpc.104.025395

Yoshimoto, K., Jikumaru, Y., Kamiya, Y., Kusano, M., Consonni, C., Panstruga, R., et al. (2009). Autophagy negatively regulates cell death by controlling NPR1dependent salicylic acid signaling during senescence and the innate immune response in Arabidopsis. Plant Cell 21, 2914-2927. doi: 10.1105/tpc.109.068635

Zaffagnini, G., and Martens, S. (2016). Mechanisms of selective autophagy. J. Mol. Biol. 428, 1714-1724. doi: 10.1016/j.jmb.2016.02.004

Zhang, K. W., and Gan, S. S. (2012). An abscisic acid-AtNAP transcription factorSAG113 protein phosphatase $2 \mathrm{C}$ regulatory chain for controlling dehydration in senescing Arabidopsis leaves. Plant Physiol. 158, 961-969. doi: 10.1104/pp. 111.190876

Zhuang, X. H., Chung, K. P., Cui, Y., Lin, W. L., Gao, C. J., Kang, B. H., et al. (2017). ATG9 regulates autophagosome progression from the endoplasmic reticulum in Arabidopsis. Proc. Natl. Acad. Sci. U.S.A. 114, E426-E435. doi: 10.1073/pnas. 1616299114

Zhuang, X. H., Chung, K. P., and Jiang, L. W. (2016). Origin of the autophagosomal membrane in plants. Front. Plant Sci. 7:1655. doi: 10.3389/fpls.2016.01655

Zhuang, X. H., Chung, K. P., Luo, M. Q., and Jiang, L. W. (2018). Autophagosome biogenesis and the endoplasmic reticulum: a plant perspective. Trends Plant Sci. 23, 677-692. doi: 10.1016/j.tplants.2018.05.002

Zhuang, X. H., Wang, H., Lam, S. K., Gao, C. J., Wang, X. F., Cai, Y., et al. (2013). A BAR-domain protein SH3P2, which binds to phosphatidylinositol 3phosphate and ATG8, regulates autophagosome formation in Arabidopsis. Plant Cell 25, 4596-4615. doi: 10.1105/tpc.113.118307

Conflict of Interest Statement: The authors declare that the research was conducted in the absence of any commercial or financial relationships that could be construed as a potential conflict of interest.

Copyright (c) 2019 Zhuang and Jiang. This is an open-access article distributed under the terms of the Creative Commons Attribution License (CC BY). The use, distribution or reproduction in other forums is permitted, provided the original author(s) and the copyright owner(s) are credited and that the original publication in this journal is cited, in accordance with accepted academic practice. No use, distribution or reproduction is permitted which does not comply with these terms. 